\title{
Retraction
}

\section{Denitrosylation of HDAC2 by targeting Nrf2 restores glucocorticosteroid sensitivity in macrophages from COPD patients}

Deepti Malhotra, Rajesh K. Thimmulappa, Nicolas Mercado, Kazuhiro Ito, Ponvijay Kombairaju, Sarvesh Kumar, Jinfang Ma, David Feller-Kopman, Robert Wise, Peter Barnes, and Shyam Biswal

Original citation: J Clin Invest. 2011;121(11):4289-4302. doi:10.1172/JCI45144.

Citation for this retraction: J Clin Invest. 2014;124(12):5521. doi:10.1172/JCI79606.

The JCI, with the agreement of the corresponding authors and coauthors, is retracting the article "Denitrosylation of HDAC2 by targeting Nrf2 restores glucocorticosteroid sensitivity in macrophages from COPD patients.” The IP: streptavidin/IB: anti-HDAC2 immunoblot panel in Figure 3B was recently identified as an inverted image of the right four lanes of the anti-H4 acetyl CHIP gel presented in Figure 2A. Additionally, the IP: anti-streptavidin/IB: anti-DDK immunoblot presented in Supplemental Figure 3A was identified as an inverted image of the left four lanes of the anti-H4 acetyl CHIP gel presented in Figure 2A. Further, in Figure 9B, the incorrect anti-HDAC2 immunoblot was presented. The authors sincerely apologize for any misinterpretation of the data as a result of these errors.

\section{Erratum}

\section{$\alpha$-Intercalated cells defend the urinary system from bacterial infection}

Neal Paragas, Ritwij Kulkarni, Max Werth, Kai M. Schmidt-Ott, Catherine Forster, Rong Deng, Qingyin Zhang, Eugenia Singer, Alexander D. Klose, Tian Huai Shen, Kevin P. Francis, Sunetra Ray, Soundarapandian Vijayakumar, Samuel Seward, Mary E. Bovino, Katherine Xu, Yared Takabe, Fábio E. Amaral, Sumit Mohan, Rebecca Wax, Kaitlyn Corbin, Simone Sanna-Cherchi, Kiyoshi Mori, Lynne Johnson, Thomas Nickolas, Vivette D’Agati, Chyuan-Sheng Lin, Andong Qiu, Qais Al-Awqati, Adam J. Ratner, and Jonathan Barasch

Original citation: J Clin Invest. 2014;124(7):2963-2976. doi:10.1172/JCI71630.

Citation for this erratum: J Clin Invest. 2014;124(12):5521. doi:10.1172/JCI79744.

There were errors in Table 1 and Figure 9D. In Table 1, the correct concentration for LCN2 is 1.80 mM. In Figure 9D, the red and blue bars were mislabeled. The correct figure panel appears below.

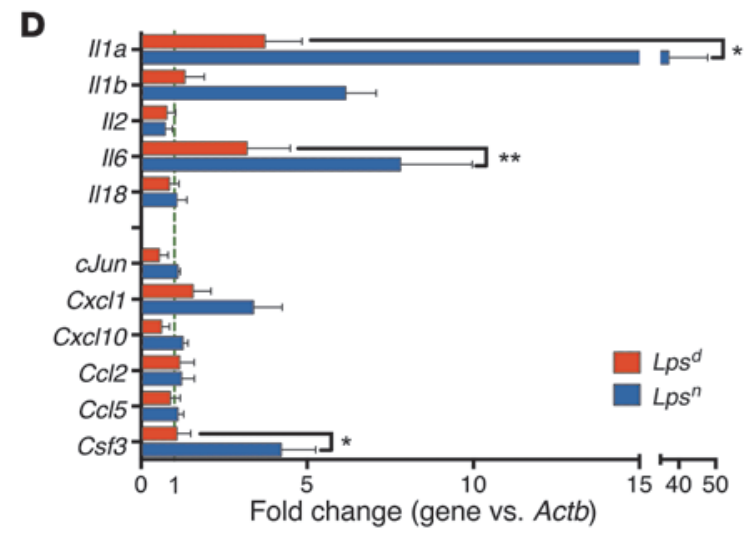

The JCI regrets the errors. 